\title{
Termorretificação e Colorimetria da Madeira de Eucalyptus grandis
}

\author{
Antônio José Vinha Zanuncio ${ }^{1}$, Elias de Sá Farias ${ }^{2}$, Teodorico Alves da Silveira ${ }^{2}$ \\ ${ }^{1}$ Departamento de Engenharia Florestal, Universidade Federal de Viçosa - UFV, Viçosa/MG, Brasil \\ ${ }^{2}$ Departamento de Ciências Florestais, Universidade Federal de Lavras - UFLA, Lavras/MG, Brasil
}

\section{RESUMO}

A termorretificação pode ser utilizada na alteração da cor da madeira, tornado-a semelhante às madeiras tropicais de maior valor agregado. O objetivo deste trabalho foi avaliar as alterações colorimétricas na madeira de Eucalyptus grandis submetida a $140,170,200$ e $230{ }^{\circ} \mathrm{C}$, por três, cinco e sete horas. Corpos de prova de $2 \times 2 \times 2 \mathrm{~cm}$ foram secos em estufa e termorretificados para avaliação de claridade $(\mathrm{L})$, matriz vermelho $\left(\mathrm{a}^{*}\right)$, matriz amarelo $\left(\mathrm{b}^{*}\right)$, saturação $(\mathrm{C})$ e ângulo de tinta $(\mathrm{H})$. A claridade $\left(\mathrm{L}^{\star}\right)$ reduziu-se a partir de $140{ }^{\circ} \mathrm{C}$; a matriz vermelho $\left(\mathrm{a}^{\star}\right)$, a partir de $140{ }^{\circ} \mathrm{C}$ por cinco horas; a matriz amarelo $\left(b^{*}\right)$, a saturação e o ângulo de tinta reduziram-se a partir de $170{ }^{\circ} \mathrm{C}$. A temperatura se mostrou mais efetiva na alteração da cor do que o tempo. A termorretificação escureceu a madeira e pode ser uma alternativa para ampliação do uso do material.

Palavras-chave: ângulo de tinta, claridade, eucalipto, tratamento térmico.

\section{Heat Treatment and Color of Eucalyptus grandis Wood}

\begin{abstract}
Heat treatment can be used to change the color of timber, adding value to it. In this study, we aimed to evaluate changes in color of Eucalyptus grandis wood subjected to 140, 170, 200, and $230{ }^{\circ} \mathrm{C}$ for three, five, and seven hours. Samples of $2 \times 2 \times 2 \mathrm{~cm}$ were dried and heat treated to evaluate lightness (L), $\mathrm{a}^{*}$ coordinate (green-red coordinate), $\mathrm{b}^{*}$ coordinate (blue-yellow coordinate), saturation $(\mathrm{C})$, and tonality angle $(\mathrm{H})$. Lightness $\left(\mathrm{L}^{*}\right)$ decreased as from $140^{\circ} \mathrm{C}$. $\mathrm{a}^{*}$ coordinate (green-red coordinate) decreased as from $140^{\circ} \mathrm{C}$ for five hours. $\mathrm{b}^{*}$ coordinate (blueyellow coordinate), saturation $(\mathrm{C})$, and tonality angle $(\mathrm{H})$, saturation, and ink angle reduced as from $170^{\circ} \mathrm{C}$. Temperature was more effective than time to change the color of timber. Heat treatment darkened the wood and can be an alternative to add value to this material.
\end{abstract}

Keywords: tonality angle, lightness, eucalyptus, heat treatment. 


\section{INTRODUÇÃO}

As propriedades colorimétricas das madeiras das florestas tropicais são desejadas para fabricação de móveis. Contudo, a exploração não sustentável destas florestas e o maior rigor dos órgãos de fiscalização estão aumentando o preço destes materiais ou até deixando-os inexistentes no mercado, criando um desequilíbrio entre oferta e procura.

A adoção de florestas plantadas de rápido crescimento está sendo utilizada para substituição da madeira nativa. Corresponderam a 6,5 milhões de hectares no Brasil, sendo 74,8\% desse total composto por florestas do gênero Eucalyptus (ABRAF, 2012). Entre as principais espécies, destacam-se: E. grandis, E. camaldulensis, E. urophylla, E. robusta, E. saligna, E. tereticornis e E. globulus (BRACELPA, 2012). Entretanto, estas espécies apresentam madeira de cor clara, dificultando a sua inserção em alguns mercados.

O processo de termorretificação pode alterar a cor da madeira de Eucalyptus, fazendo com que esta se aproxime das madeiras de maior valor de mercado. Este processo é muito utilizado nos EUA e na Europa, e vem ganhando força em virtude do seu caráter pouco poluente. No Brasil, o comércio de madeira termorretificada é inexpressivo.

Além das alterações na cor, a termorretificação melhora o comportamento higroscópico, a estabilidade dimensional e a resistência biológica da madeira (Korkut, 2012; Almeida et al., 2009; Ratnasingam \& Ioras, 2012; Bal \& Bektas, 2012), em detrimento das propriedades mecânicas (Garcia et al., 2012; Dundar et al., 2012; Cademartori et al., 2012).

O sistema CIELAB, de 1976, é o mais utilizado na avaliação colorimétrica de materiais (Pincelli et al., 2012). Neste sistema, L indica a claridade e varia entre 0 e 100, em que zero representa o negro e 100, o branco. $\mathrm{O}^{*}$ indica a matriz vermelho, em que o número positivo indica o vermelho e o negativo, $o$ verde. $\mathrm{O} \mathrm{b}^{*}$ indica a matriz amarelo, sendo que o número positivo indica o amarelo e o negativo, $\mathrm{o}$ azul. A saturação (C) indica a pureza da cor, enquanto o ângulo de tinta $(\mathrm{H})$ indica a dominância de alguma tonalidade na cor.

A termorretificação altera a cor da madeira, possibilitando o uso mais amplo da mesma. O objetivo deste trabalho foi avaliar o tratamento térmico da madeira nas suas propriedades colorimétricas.

\section{MATERIAL E MÉTODOS}

Três árvores de Eucalyptus grandis com 15 anos de idade foram utilizadas, por esta ser uma das espécies mais utilizadas no Brasil, e nessa idade, por ser a recomendada para uso como madeira sólida.

Após o abate das árvores, uma tora de um metro foi retirada à altura de 1,3 metro acima do solo; as mesmas foram cortadas em tábuas e secas ao ar livre, até atingiram umidade de equilíbrio. Em seguida, utilizaram-se as tábuas para confecção de corpos de prova de $2 \times 2 \times 2 \mathrm{~cm}$, que foram secos em estufa por $24 \mathrm{~h}$ à temperatura de $105^{\circ} \mathrm{C}$, para obtenção de madeira anidra. $\mathrm{O}$ tratamento térmico ocorreu em estufa a $140,170,200$ e $230{ }^{\circ} \mathrm{C}$ por três, cinco e sete horas, sob pressão atmosférica e presença de ar. As amostras foram retiradas da estufa após resfriamento até $25^{\circ} \mathrm{C}$ e cada tratamento teve 60 repetições.

A análise colorimétrica foi feita na superfície longitudinal das amostras com espectrofotômetro Konica Minolta CM-2500D. Os parâmetros colorimétricos avaliados foram claridade (L), coordenada vermelho-verde ou matriz vermelho $\left(\mathrm{a}^{*}\right)$ e coordenada azul-amarelo ou matriz amarelo $\left(b^{*}\right)$, baseados no Sistema de cor CIELAB 1976.

A saturação (C) foi calculado pela Equação 1.

$\mathrm{C}=\left[\left(\mathrm{a}^{*}\right)^{2}+\left(\mathrm{b}^{*}\right)^{2}\right]^{0,5}$

em que: $\mathrm{C}=$ saturação; $\mathrm{a}^{*}=$ matriz vermelho; $\mathrm{b}^{*}=$ matriz amarelo.

$\mathrm{O}$ ângulo de tinta $(\mathrm{H})$ foi determinado conforme Equação 2.

$\mathrm{H}=\operatorname{arctang}\left(\mathrm{b}^{*} / \mathrm{a}^{*}\right)$

em que: $b^{\star}=$ matriz amarelo; $a^{\star}=$ matriz vermelho; $\mathrm{H}=$ ângulo de tinta.

O contraste entre as médias foi determinado pelo teste de Scott-Knott a 5\% de nível de significância.

\section{RESULTADOS E DISCUSSÃO}

A claridade variou entre 65,3 e 32,3. Notou-se que à medida que o processo de termorretificação se intensificava, os valores diminuíam, evidenciando o escurecimento da madeira (Figura 1).

A temperatura se mostrou mais efetiva na redução da claridade do que o tempo. O tratamento a 
$140{ }^{\circ} \mathrm{C}$ por três horas reduziu a claridade em $12,25 \%$ e o aumento do tempo de três para sete horas reduziu a claridade em $16,23 \%$. A temperatura de $170{ }^{\circ} \mathrm{C}$ durante três horas diminuiu a claridade em $21,13 \%$ e o aumento de quatro horas no tratamento térmico diminuiu a claridade em $30,16 \%$. O incremento da temperatura para $200{ }^{\circ} \mathrm{C}$ reduziu a claridade em $42,11 \%$ e $46,71 \%$, nos tempos de três e sete horas, respectivamente (Figura 1).

Na temperatura de $230{ }^{\circ} \mathrm{C}$, os tratamentos com três, cinco e sete horas se mostraram iguais. Neste caso, a degradação dos extrativos e hemiceluloses, que tornam a madeira escura, é mais acelerada, enquanto que, nas temperaturas inferiores, a degradação destes mesmos componentes é mais demorada, justificando a diferença entre os tratamentos com diferentes durações e mesma temperatura a 140,170 e $200{ }^{\circ} \mathrm{C}$ (Figura 1).

A matriz vermelho $\left(a^{*}\right)$ variou entre 12,6 e 3,7 . Observou-se que houve diminuição dos valores com o aumento da intensidade dos tratamentos (Figura 2). A redução destes valores é devido à volatização dos extrativos polares, sendo a maior queda na temperatura de $200{ }^{\circ} \mathrm{C}$, faixa correspondente à decomposição desta classe de extrativos, entre 130 e $250{ }^{\circ} \mathrm{C}$ (Mészáros et al., 2007). Pincelli et al. (2012) relataram queda de 12,8 para 10,5, enquanto Moura \& Brito (2011) relataram queda de 18,51 para 9,76 , sendo ambos os estudos para Eucalytus grandis submetido a $180^{\circ} \mathrm{C}$.

A matriz amarelo $\left(b^{*}\right)$ não reduziu na madeira tratada a $140^{\circ} \mathrm{C}$ (Figura 3). A maior redução ocorreu com $170^{\circ} \mathrm{C}$, de $32,9 \%$. Houve diferença entre o tempo,

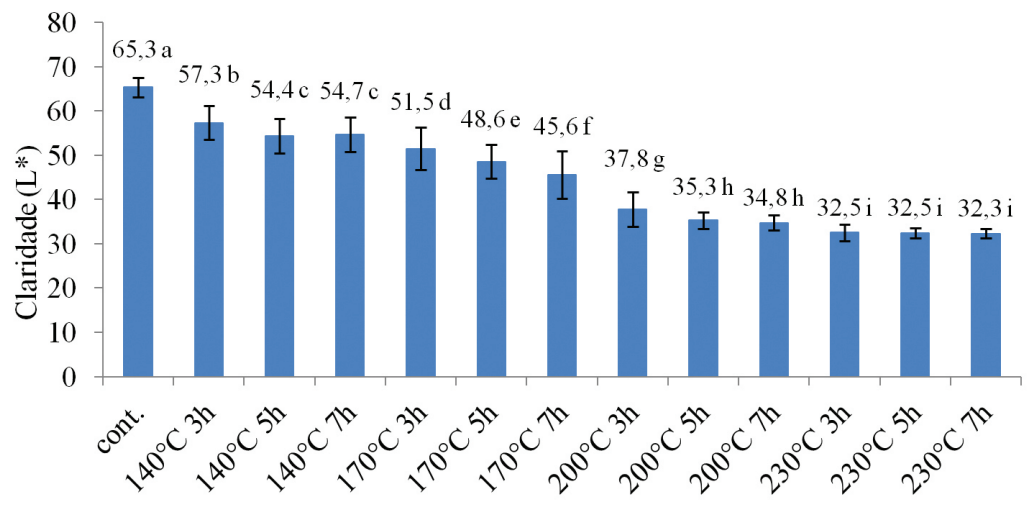

Figura 1. Claridade $\left(\mathrm{L}^{*}\right)$ da madeira de Eucalyptus grandis termorretificada. Médias seguidas por mesma letra pertencem a um mesmo grupo pelo teste Scott-Knott, a 5\% de significância.

Figure 1. Lightness ( $\mathrm{L}^{\star}$ ) of Eucalyptus grandis heat treated. Means followed by the same letter do not differ by ScottKnott test at $5 \%$.

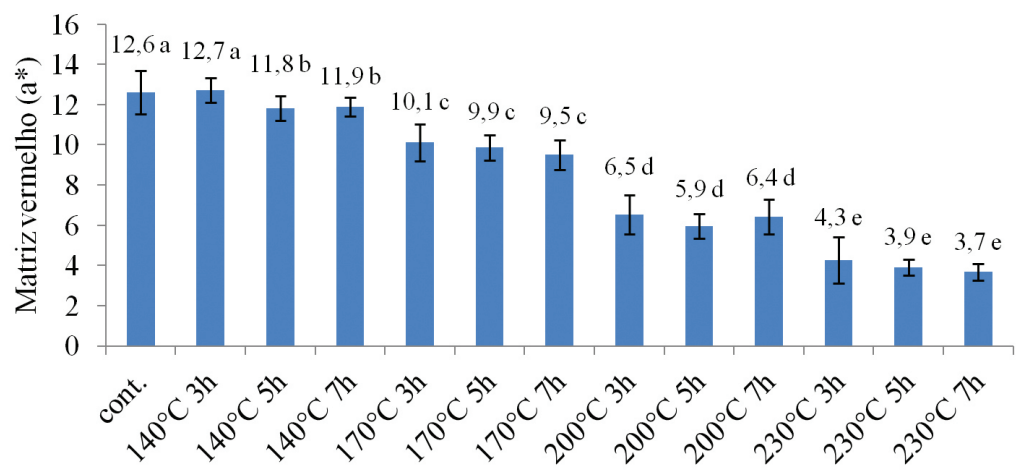

Figura 2. Matriz vermelho $\left(\mathrm{a}^{\star}\right)$ da madeira de Eucalyptus grandis termorretificada. Médias seguidas de mesma letra pertencem a um mesmo grupo pelo teste Scott-Knott a 5\% de nível de significância.

Figure 2. Green-red coordinate $\left(\mathrm{a}^{*}\right)$ of Eucalyptus grandis heat treated. Means followed by the same letter do not differ by Scott-Knott test at $5 \%$. 
para os tratamentos na mesma temperatura, para 170,200 e $230^{\circ} \mathrm{C}$. Compostos cromóforos associados às ligninas e aos extrativos conferem a cor amarelada à madeira; entretanto, o tratamento térmico degrada estes compostos reduzindo a matriz amarelo $\left(b^{*}\right)$ (Pincelli et al., 2012). Cademartori et al. (2013) relataram queda na matriz amarelo $\left(b^{*}\right)$ de 20,57 para 17,69 e 10,93, para madeira de Eucalyptus grandis a $180{ }^{\circ} \mathrm{C}$ e $240{ }^{\circ} \mathrm{C}$ por quatro horas, respectivamente. Moura \& Brito (2011), por sua vez, relataram queda na matriz amarelo $\left(b^{*}\right)$ de 21,74 para 18,20 e 14,34 , para Eucalyptus grandis submetido a $160^{\circ} \mathrm{C}$ e $180^{\circ} \mathrm{C}$, respectivamente.

A saturação apresentou comportamento semelhante à matriz amarelo $\left(b^{*}\right)$ (Figura 4). Não houve redução a $140{ }^{\circ} \mathrm{C}$, seguida de queda nas temperaturas de 170,200 e $230^{\circ} \mathrm{C}$. Houve diferença entre o tempo, para mesma temperatura, nestas três faixas. Os valores de saturação (C) dependem da matriz vermelho $\left(a^{*}\right)$ e amarelo $\left(b^{\star}\right)$. Na medida em que os valores de $b^{\star}$ para madeira são em geral maiores que os de $\mathrm{a}^{*}$, assume-se que a matriz amarelo $\left(b^{*}\right)$ tem mais influência sobre a saturação, o que explica o comportamento semelhante entre $b^{\star}$ e C.

Para o ângulo de tinta $(\mathrm{H})$, não houve redução nos tratamentos a $140{ }^{\circ} \mathrm{C}$. Os tratamentos a 170 e $200{ }^{\circ} \mathrm{C}$ se mostraram iguais, com redução para os tratamentos a $230{ }^{\circ} \mathrm{C}$ (Figura 5). O ângulo de tinta $(\mathrm{H})$ é resultante das matrizes vermelho $\mathrm{a}^{*} \mathrm{e}$ amarelo $b^{*}$. Desse modo, o ângulo de tinta mostrou comportamento parecido com a matriz amarelo devido ao seu maior valor em relação à matriz

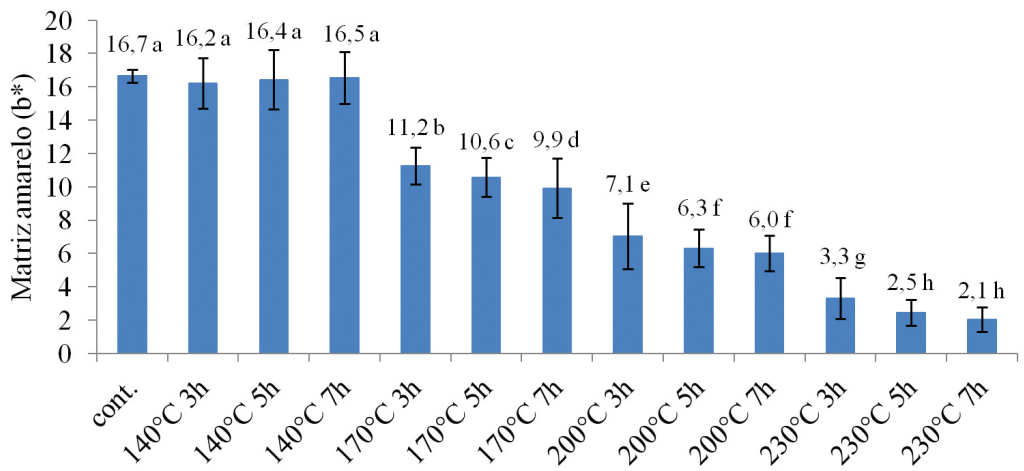

Figura 3. Matriz amarelo $\left(b^{\star}\right)$ da madeira de Eucalyptus grandis termorretificada. Médias seguidas de mesma letra pertencem a um mesmo grupo pelo teste Scott-Knott a 5\% de nível de significância.

Figure 3. Blue-yellow coordinate $\left(\mathrm{b}^{*}\right)$ of Eucalyptus grandis heat treated. Means followed by the same letter do not differ by Scott-Knott test at $5 \%$.

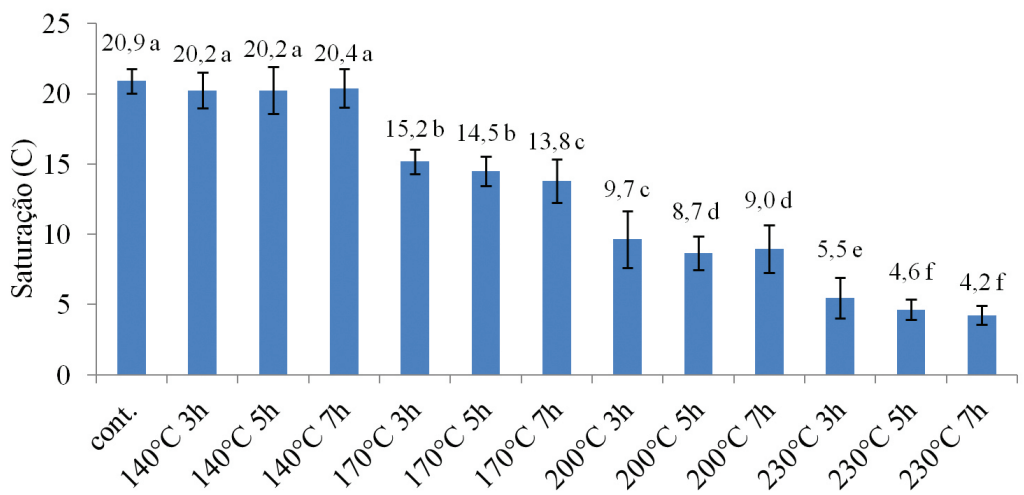

Figura 4. Saturação (C) da madeira de Eucalyptus grandis termorretificada. Médias seguidas de mesma letra pertencem a um mesmo grupo pelo teste Scott-Knott a 5\% de nível de significância.

Figure 4. Saturation (C) of Eucalyptus grandis heat treated. Means followed by the same letter do not differ by ScottKnott test at 5\%. 


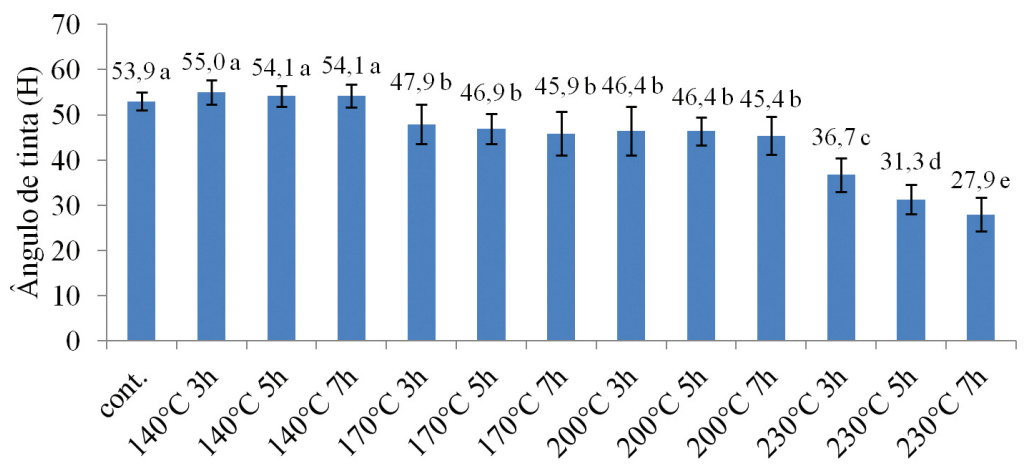

Figura 5. Ângulo de Tinta $(\mathrm{H})$ da madeira de Eucalyptus grandis termorretificada. Médias seguidas de mesma letra pertencem a um mesmo grupo pelo teste Scott-Knott a 5\% de nível de significância.

Figure 5. Tonality angle (H) of Eucalyptus grandis heat treated. Means followed by the same letter do not differ by Scott-Knott test at $5 \%$.

vermelho. Pincelli et al. (2012) relataram tendência inversa ao deste trabalho, com aumento de 55,8 para 58,7, enquanto Cademartori et al. (2013) relataram redução de 66,15 para 62,21 , sendo ambos os estudos para Eucalyptus grandis a $180^{\circ} \mathrm{C}$.

Alterações colorimétricas na madeira na etapa de secagem não são desejadas. Contudo, o escurecimento causado pela termorretificação na madeira pode agregar valor e ampliar seu uso. Além disso, a coloração da madeira através de tintas emite tolueno e xileno, nocivos à saúde humana e ao meio ambiente (Korkut, 2012).

\section{CONCLUSÕES}

A claridade $\left(\mathrm{L}^{\star}\right)$ reduziu a partir de $140{ }^{\circ} \mathrm{C}$, evidenciando o escurecimento da madeira, havendo diferença entre tempos, na mesma temperatura, para $140{ }^{\circ} \mathrm{C}$ e $170{ }^{\circ} \mathrm{C}$ e $200{ }^{\circ} \mathrm{C}$. A matriz vermelho $\left(\mathrm{a}^{\star}\right)$ reduziu a partir de $140{ }^{\circ} \mathrm{C}$ por cinco horas. A matriz amarelo $\left(b^{*}\right)$, a saturação e o ângulo de tinta reduziram a partir de $170^{\circ} \mathrm{C}$.

A termorretificação é uma alternativa para alteração das cores da madeira, visando ao seu escurecimento e à queda das matrizes vermelho $\left(a^{*}\right)$ e amarelo $\left(b^{\star}\right)$. Estas alterações podem agregar valor à madeira e ampliar sua utilização.

\section{AGRADECIMENTOS}

À Fundação de Amparo à Pesquisa do Estado de Minas Gerais (FAPEMIG), à Coordenação de Aperfeiçoamento de Pessoal de Nível Superior (CAPES) e ao Conselho Nacional de Desenvolvimento Científico e Tecnológico (CNPq), pelo apoio financeiro.

\section{STATUS DA SUBMISSÃO}

Recebido: $12 / 08 / 2013$

Aceito: 03/12/2013

Publicado: 31/02/2014

\section{AUTOR(ES) PARA CORRESPONDÊNCIA}

\section{Antonio José Vinha Zanuncio}

Departamento de Engenharia Florestal, Universidade Fedral de Viçosa - UFV, CEP 37570-000, Viçosa, MG, Brasil e-mail: ajvzanuncio@yahoo.com.br

\section{REFERÊNCIAS}

Almeida G, Brito JO, Perre P. Changes in wood-water relationship due to heat treatment assessed on microsamples of three Eucalyptus species. Holzforschung 2009; 63(1): 80-88. http://dx.doi.org/10.1515/HF.2009.026

Associação Brasileira de Celulose e Papel - BRACELPA. Relatório estatístico 2011. [cited 2012 jul 27]. Available from: http://www.bracelpa.org.br.

Associação Brasileira de Produtores de Florestas Plantadas - ABRAF. Anuário estatístico da ABRAF: ano base 2011. Brasília, 2012. p. 145.

Bal BC, Bektas I. The effects of heat treatment on physical properties of juvenile wood and mature wood 
of Eucalyptus grandis. Bioresources 2012; 7(4): 51175127.

Cademartori PHG, Schneid E, Gatto DA, Beltrame R, Stangerlin DM. Modification of Static Bending Strength Properties of Eucalyptus grandis Heat-Treated Wood. Materials Research 2012; 15(6): 922-927. http://dx.doi. org/10.1590/S1516-14392012005000136

Cademartori PHG, Schneid E, Gatto DA, Stangerlin DM, Beltrame R. Thermal modification of Eucalyptus grandis wood: variation of colorimetric parameters. Maderas. Ciencia y tecnología 2013; 15(1): 57-64.

Dundar T, Buyuksari U, Avci E, Akkiliç H. Effect of heat treatment on the physical and mechanical properties of compression and opposite wood of black pine. Bioresources 2012; 7(4): 5009-5018.

Garcia RA, Carvalho AM, Latorraca JVF, Matos JM, Santos VA, Silva RFM. Nondestructive evaluation of heat-treated Eucalyptus grandis Hill ex Maiden wood using stress wave method. Wood Science and Technology 2012; 46(3): 41-52. http://dx.doi.org/10.1007/s00226010-0387-6
Korkut S. Performance of three thermally treated tropical wood species commonly used in Turkey. Industrial Crops and Products 2012; 36(1): 355-362. http://dx.doi.org/10.1016/j.indcrop.2011.10.004

Mészáros E, Jakab E, Várhegyi G. TG/MS, Py-GC/ MS and THM-GC/MS study of the composition and thermal behavior of extractive components of Robinia pseudoacacia. Journal of Analytical and Applied Pyrolysis 2007; 79(1): 61-70. http://dx.doi.org/10.1016/j. jaap.2006.12.007

Moura LF, Brito JO. Efeito da termorretificação sobre as propriedades colorimétricas das madeiras de Eucalyptus grandis e Pinus caribaea var. hondurensis. Scientia Forestalis 2011; 39(89): 069-076.

Pincelli ALPSM, De Moura LM, Brito JO. Effect of thermal rectification on colors of Eucalyptus Saligna and Pinus Caribaea woods. Maderas. Ciencia y tecnología 2012; 14(2): 239-248

Ratnasingam J, Ioras F. Effect of heat treatment on the machining and other properties of rubberwood. European Journal of Wood and Wood Products 2012; 70(5): 759-761. http://dx.doi.org/10.1007/s00107-0110587-2 